AperTO - Archivio Istituzionale Open Access dell'Università di Torino

\title{
NESCOND: an Implementation of Nested Sequent Calculi for Conditional Logics
}

\section{This is the author's manuscript}

Original Citation:

\section{Availability:}

This version is available http://hdl.handle.net/2318/152376

since 2016-06-28T15:44:56Z

Publisher:

Demri Stéphane, Kapur Deepak, Weidenbach Christoph

Published version:

DOI:10.1007/978-3-319-08587-6-39

Terms of use:

Open Access

Anyone can freely access the full text of works made available as "Open Access". Works made available under a Creative Commons license can be used according to the terms and conditions of said license. Use of all other works requires consent of the right holder (author or publisher) if not exempted from copyright protection by the applicable law. 


\title{
NESCOND: An Implementation of Nested Sequent Calculi for Conditional Logics
}

\author{
Nicola Olivetti ${ }^{1}$ and Gian Luca Pozzato ${ }^{2}$ \\ 1 Aix-Marseille Université, CNRS, LSIS UMR 7296 - France \\ nicola.olivetti@univ-amu.fr \\ 2 Dipartimento di Informatica - Universitá di Torino - Italy \\ gianluca.pozzato@unito.it
}

\begin{abstract}
We present NESCOND, a theorem prover for normal conditional logics. NESCOND implements some recently introduced NESted sequent calculi for propositional CONDitional logics CK and some of its significant extensions with axioms ID, MP and CEM. It also deals with the flat fragment of CK+CSO+ID, which corresponds to the logic $\mathbf{C}$ introduced by Kraus, Lehmann and Magidor. NESCOND is inspired by the methodology of lean $T^{A} P$ and it is implemented in Prolog. The paper shows some experimental results, witnessing that the performances of NESCOND are promising. The program NESCOND, as well as all the Prolog source files, are available at http://www.di.unito.it/ \%7Epozzato/nescond/.
\end{abstract}

\section{Introduction}

Conditional logics are extensions of classical logic by a conditional operator $\Rightarrow$. They have a long history [10, 11], and recently they have found an interest in several fields of AI and knowledge representation. Just to mention a few (see [2] for a complete bibliography), they have been used to reason about prototypical properties, to model belief change, to reason about access control policies, to formalize epistemic change in a multi-agent setting. Conditional logics can also provide an axiomatic foundation of nonmonotonic reasoning [9]: here a conditional $A \Rightarrow B$ is read "normally, if $A$ then B'.

In previous works [1, 2] we have introduced nested sequent calculi, called $\mathcal{N} S$, for propositional conditional logics. Nested sequent calculi [4-6, 8] are a natural generalization of ordinary sequent calculi where sequents are allowed to occur within sequents. However, a nested sequent always corresponds to a formula of the language, so that we can think of the rules as operating "inside a formula", combining subformulas rather than just combining outer occurrences of formulas as in ordinary sequent calculi. The basic normal conditional logic CK and its extensions with ID, MP and CEM are considered, as well as the cumulative logic $\mathbf{C}$ introduced in [9] which corresponds to the flat fragment (i.e., without nested conditionals) of the conditional logic CK+CSO+ID.

Here we describe an implementation of $\mathcal{N} S$ in Prolog. The program, called NESCOND, gives a PSPACE decision procedure for the respective logics, and it is inspired by the methodology of $\operatorname{lean} T^{A} P$ [3]. The idea is that each axiom or rule of 
the nested sequent calculi is implemented by a Prolog clause of the program. The resulting code is therefore simple and compact: the implementation of NESCOND for CK consists of only 6 predicates, 24 clauses and 34 lines of code. We provide experimental results by comparing NESCOND with CondLean [12] and GOALDUCK [13]. Performances of NESCOND are promising, and show that nested sequent calculi are not only a proof theoretical tool, but they can be the basis of efficient theorem proving for conditional logics.

\section{Conditional Logics and Their Nested Sequent Calculi}

We consider a propositional conditional language $\mathcal{L}$ over a set $A T M$ of propositional variables. Formulas of $\mathcal{L}$ are built as usual: $\perp$, $\top$ and the propositional variables of $A T M$ are atomic formulas; if $A$ and $B$ are formulas, then $\neg A$ and $A \circ B$ are complex formulas, where $\circ \in\{\wedge, \vee, \rightarrow, \Rightarrow\}$. We adopt the selection function semantics. We consider a non-empty set of possible worlds $\mathcal{W}$. Intuitively, the selection function $f$ selects, for a world $w$ and a formula $A$, the set of worlds of $\mathcal{W}$ which are closest to $w$ given the information $A$. A conditional formula $A \Rightarrow B$ holds in a world $w$ if the formula $B$ holds in all the worlds selected by $f$ for $w$ and $A$.

Definition 1 (Selection function semantics). A model is a triple $\mathcal{M}=\langle\mathcal{W}, f,[]\rangle$ where $\mathcal{W}$ is a non empty set of worlds, $f: \mathcal{W} \times 2^{\mathcal{W}} \longmapsto 2^{\mathcal{W}}$ is the selection function, and [ ] is the evaluation function, which assigns to an atom $P \in A T M$ the set of worlds where $P$ is true, and is extended to boolean formulas as follows: $[\top]=\mathcal{W} ;[\perp]=\emptyset$; $[\neg A]=\mathcal{W}-[A] ;[A \wedge B]=[A] \cap[B] ;[A \vee B]=[A] \cup[B] ;[A \rightarrow B]=[B] \cup(\mathcal{W}-[A]) ;$ $[A \Rightarrow B]=\{w \in \mathcal{W} \mid f(w,[A]) \subseteq[B]\}$. A formula $F \in \mathcal{L}$ is valid in a model $\mathcal{M}=\langle\mathcal{W}, f,[]\rangle$, and we write $\mathcal{M} \models F$, if $[F]=\mathcal{W}$. A formula $F \in \mathcal{L}$ is valid, and we write $\models F$, if it is valid in every model, that is to say $\mathcal{M} \models F$ for every $\mathcal{M}$.

The semantics above characterizes the basic conditional system, called CK, where no specific properties of the selection function are assumed. An axiomatization of CK is given by $(\vdash$ denotes provability in the axiom system):

- any axiomatization of the classical propositional calculus

- If $\vdash A$ and $\vdash A \rightarrow B$, then $\vdash B$

- If $\vdash A \leftrightarrow B$ then $\vdash(A \Rightarrow C) \leftrightarrow(B \Rightarrow C)$

(RCEA)

- If $\vdash\left(A_{1} \wedge \cdots \wedge A_{n}\right) \rightarrow B$ then $\vdash\left(C \Rightarrow A_{1} \wedge \cdots \wedge C \Rightarrow A_{n}\right) \rightarrow(C \Rightarrow B) \quad$ (RCK)

Moreover, we consider the following standard extensions of the basic system CK:

\begin{tabular}{|l|l|l|}
\hline SYSTEM & AXIOM & MODEL CONDITION \\
ID & $A \Rightarrow A$ & $f(w,[A]) \subseteq[A]$ \\
CEM & $(A \Rightarrow B) \vee(A \Rightarrow \neg B)$ & $|f(w,[A])| \leq 1$ \\
MP & $(A \Rightarrow B) \rightarrow(A \rightarrow B)$ & $w \in[A]$ implies $w \in f(w,[A])$ \\
CSO & $(A \Rightarrow B) \wedge(B \Rightarrow A) \rightarrow((A \Rightarrow C) \rightarrow(B \Rightarrow C))$ & $f(w,[A]) \sqsubseteq[B]$ and $f(w,[B]) \sqsubseteq[A]$ implies $f(w,[A])=f(w,[B])$
\end{tabular}

In Figure 1 we present nested sequent calculi $\mathcal{N} S$, where $S$ is an abbreviation for $\mathrm{CK}\{+\mathrm{X}\}$, and $\mathrm{X}=\{\mathrm{CEM}, \mathrm{ID}, \mathrm{MP}, \mathrm{ID}+\mathrm{MP}, \mathrm{CEM}+\mathrm{ID}\}$. A nested sequent $\Gamma$ is defined inductively as follows: a formula of $\mathcal{L}$ is a nested sequent; if $A$ is a formula and $\Gamma$ is a nested sequent, then $[A: \Gamma]$ is a nested sequent; a finite multiset of nested sequents is a nested sequent. A nested sequent can be displayed as 


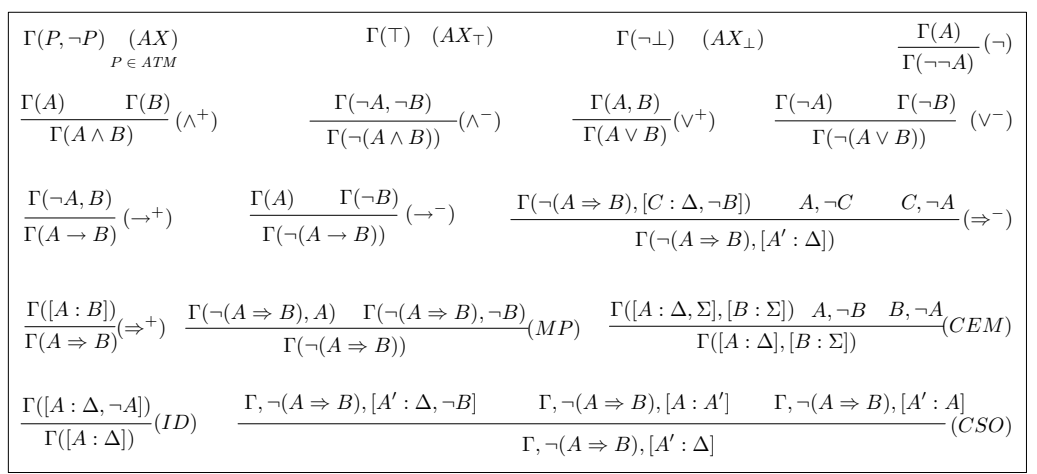

Fig. 1. The nested sequent calculi $\mathcal{N} S$.

$$
A_{1}, \ldots, A_{m},\left[B_{1}: \Gamma_{1}\right], \ldots,\left[B_{n}: \Gamma_{n}\right],
$$

where $n, m \geq 0, A_{1}, \ldots, A_{m}, B_{1}, \ldots, B_{n}$ are formulas and $\Gamma_{1}, \ldots, \Gamma_{n}$ are nested sequents. A nested sequent can be directly interpreted as a formula by replacing "," by $\vee$ and ":" by $\Rightarrow$, i.e. the interpretation of $A_{1}, \ldots, A_{m},\left[B_{1}: \Gamma_{1}\right], \ldots,\left[B_{n}: \Gamma_{n}\right]$ is inductively defined by the formula $\mathcal{F}(\Gamma)=A_{1} \vee \ldots \vee A_{m} \vee\left(B_{1} \Rightarrow \mathcal{F}\left(\Gamma_{1}\right)\right) \vee \ldots \vee\left(B_{n} \Rightarrow\right.$ $\left.\mathcal{F}\left(\Gamma_{n}\right)\right)$.

We have also provided nested sequent calculi for the flat fragment, i.e. without nested conditionals, of $\mathrm{CK}+\mathrm{CSO}+\mathrm{ID}$, corresponding to KLM logic $\mathbf{C}$ [9]. The rules of the calculus, called $\mathcal{N} \mathbf{C}_{\mathrm{KLM}}$, are those ones of $\mathcal{N C K}+\mathrm{ID}$ (restricted to the flat fragment) where the rule $\left(\Rightarrow^{-}\right)$is replaced by the rule $(C S O)$.

In order to present the rules of the calculus, we need the notion of context. Intuitively a context denotes a "hole", a unique empty position, within a sequent that can be filled by a sequent. We use the symbol ( ) to denote the empty context. A context is defined inductively as follows: $\Gamma()=\Delta,()$ is a context; if $\Sigma()$ is a context $\Gamma()=\Delta,[A$ : $\Sigma()]$ is a context. Finally, we define the result of filling "the hole" of a context by a sequent. Let $\Gamma($ ) be a context and $\Delta$ be a sequent, then the sequent obtained by filling the context by $\Delta$, denoted by $\Gamma(\Delta)$ is defined as follows: if $\Gamma()=\Lambda,()$ then $\Gamma(\Delta)=\Lambda, \Delta$; if $\Gamma()=\Lambda,[A: \Sigma()]$ then $\Gamma(\Delta)=\Lambda,[A: \Sigma(\Delta)]$. The notions of derivation and of derivable sequent are defined as usual. In [1] we have shown that:

Theorem 1. The nested sequent calculi $\mathcal{N} S$ are sound and complete for the respective logics, i.e. a formula $F$ of $\mathcal{L}$ is valid in $C K+X$ if and only if it is derivable in $\mathcal{N} S$.

As usual, in order to obtain a decision procedure for the conditional logics under consideration, we have to control the application of the rules $\left(\Rightarrow^{-}\right) /(C S O),(M P),(C E M)$, and $(I D)$ that otherwise may be applied infinitely often in a backward proof search, since their principal formula is copied into the respective premise(s). We obtain a sound, complete and terminating calculus if we restrict the applications of these rules as follows [1, 2]: $\left(\Rightarrow^{-}\right)$can be applied only once to each formula $\neg(A \Rightarrow B)$ with a context $[C: \Delta]$ in each branch - the same for $(C S O)$ in the system CK+CSO+ID; $(I D)$ can be applied only once to each context $[A: \Delta]$ in each branch; $(M P)$ can be applied only once to each formula $\neg(A \Rightarrow B)$ in each branch. For systems with $(C E M)$, we need 
a more complicated mechanism: due to space limitations, we refer to [1] for this case. These results give a PSPACE decision procedure for their respective logics.

\section{Design of NESCOND}

In this section we present a Prolog implementation of the nested sequent calculi $\mathcal{N} S$. The program, called NESCOND (NESted sequent calculi for CONDitional logics), is inspired by the "lean" methodology of lean $T^{A} P$, even if it does not follow its style in a rigorous manner. The program comprises a set of clauses, each one of them implements a sequent rule or axiom of $\mathcal{N} S$. The proof search is provided for free by the mere depthfirst search mechanism of Prolog, without any additional ad hoc mechanism.

NESCOND represents a nested sequent with a Prolog list, whose elements can be either formulas F or pairs [Context, AppliedConditionals] where:

- Context is also a pair of the form [F, Gamma], where $\mathrm{F}$ is a formula of $\mathcal{L}$ and Gamma is a Prolog list representing a nested sequent;

- AppliedConditionals is a Prolog list $\left[\mathrm{A}_{-} 1=>\mathrm{B}_{-} 1, \mathrm{~A}_{-} 2=>\mathrm{B}_{-} 2, \ldots, \mathrm{A} \_\mathrm{k}=>\right.$ B_k], keeping track of the negated conditionals to which the rule $\left(\Rightarrow^{-}\right)$has been already applied by using Context in the current branch. This is used in order to implement the restriction on the application of the rule $\left(\Rightarrow^{-}\right)$in order to ensure termination.

Symbols $T$ and $\perp$ are represented by constants true and false, respectively, whereas connectives $\neg, \wedge, \vee, \rightarrow$, and $\Rightarrow$ are represented by $!, \wedge, \vee,->$, and $=>$.

As an example, the Prolog list $[p, q, !(p=>),[[p,[q \vee ! p,[[p,[p=>r]],[]]$, !r] ], [p $\Rightarrow \mathrm{q}]],[[\mathrm{q},[\mathrm{p}, ! \mathrm{p}]],[]]]$ represents the nested sequent $P, Q, \neg(P \Rightarrow Q)$, $[P: Q \vee \neg P,[P: P \Rightarrow R], \neg R],[Q: P, \neg P]$. Furthermore, the list $[\mathrm{p}=>\mathrm{q}]$ in the leftmost context is used to represent the fact that, in a backward proof search, the rule $\left(\Rightarrow^{-}\right)$has already been applied to $\neg(P \Rightarrow Q)$ by using $[P: Q \vee \neg P,[P: P \Rightarrow R], \neg R]$.

Auxiliary Predicates. In order to manipulate formulas "inside" a sequent, NESCOND makes use of the three following auxiliary predicates:

- deepMember (+Formulas, +NS) succeeds if and only if either (i) the nested sequent NS representing a nested sequent $\Gamma$ contains all the fomulas in the list Formulas or (ii) there exists a context [ [A, Delta], AppliedConditionals] in NS such that deepMember (Formulas, Delta) succeeds, that is to say there is a nested sequent occurring in NS containing all the formulas of Formulas.

- deepselect (+Formulas, +NS, -NewNS) operates exactly as deepMember, however it removes the formulas of the list Formulas by replacing them with a placeholder hole; the output term NewNS matches the resulting sequent.

- fillTheHole (+NewNS, +Formulas,-DefNS) replaces hole in NewNS with the formulas in the list Formulas. DefNS is the output term matching the result.

NESCOND for CK. The calculi $\mathcal{N} S$ are implemented by the predicate prove (+NS, -ProofTree). This predicate succeeds if and only if the nested sequent represented by the list NS is derivable. When it succeeds, the output term ProofTree matches with a representation of the derivation found by the prover. For instance, in order to prove that the formula $(A \Rightarrow(B \wedge C)) \rightarrow(A \Rightarrow B)$ is valid in CK, one queries NESCOND 
with the goal: prove $([(\mathrm{a}=>\mathrm{b} \wedge \mathrm{c})->(\mathrm{a}=>\mathrm{b})]$, ProofTree $)$. Each clause of prove implements an axiom or rule of $\mathcal{N} S$. To search a derivation of a nested sequent $\Gamma$, NESCOND proceeds as follows. First of all, if $\Gamma$ is an axiom, the goal will succeed immediately by using one of the following clauses for the axioms:

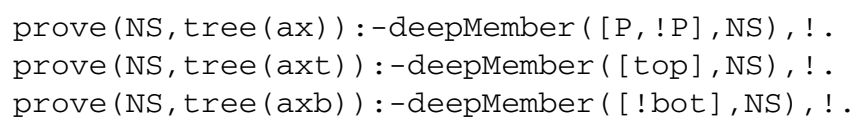

implementing $(A X),\left(A X_{\top}\right)$ and $\left(A X_{\perp}\right)$, respectively. If $\Gamma$ is not an instance of the axioms, then the first applicable rule will be chosen, e.g. if a nested sequent in $\Gamma$ contains a formula $\mathrm{A} V \mathrm{~B}$, then the clause implementing the $\left(\mathrm{V}^{+}\right)$rule will be chosen, and NESCOND will be recursively invoked on the unique premise of $\left(\mathrm{V}^{+}\right)$. NESCOND proceeds in a similar way for the other rules. The ordering of the clauses is such that the application of the branching rules is postponed as much as possible.

As an example, the clause implementing $\left(\Rightarrow^{-}\right)$is as follows:

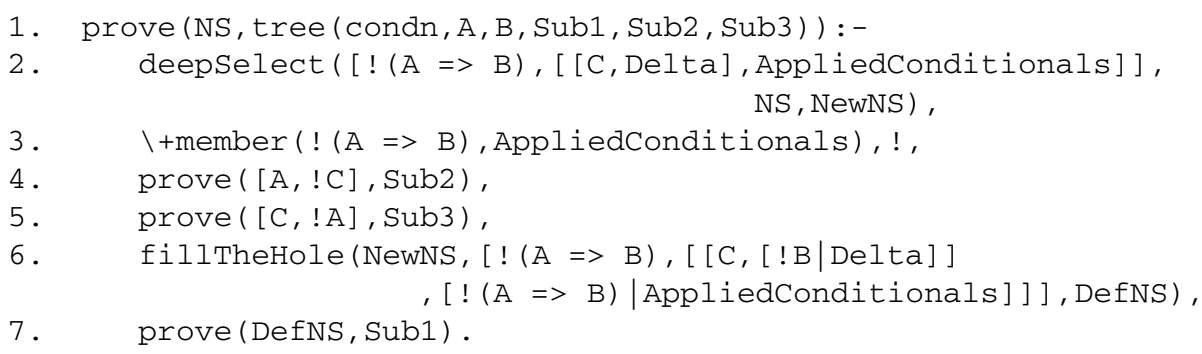

In line 2, the auxiliary predicate deepselect is invoked in order to find both a negated conditional $\neg(A \Rightarrow B)$ and a context $[C: \Delta]$ in the sequent (even in a nested subsequent). In this case, such formulas are replaced by the placeholder hole. Line 3 implements the restriction on the application of $\left(\Rightarrow^{-}\right)$in order to guarantee termination: the rule is applied only if $\neg(A \Rightarrow B)$ does not belong to the list AppliedConditionals of the selected context. In lines 4, 5 and 7, NESCOND is recursively invoked on the three premises of the rule. In line 7, NESCOND is invoked on the premise in which the context $[C: \Delta]$ is replaced by $[C: \Delta, \neg B]$. To this aim, in line 6 the auxiliary predicate fillTheHole (+NewNS, +Formulas, -DefNS) is invoked to replace the hole in NewNS, introduced by deepselect, with the negated conditional $\neg(A \Rightarrow B)$, which is copied into the premise, and the context $[C: \Delta, \neg B]$, whose list of AppliedConditionals is updated by adding the formula $\neg(A \Rightarrow B)$ itself.

NESCOND for Extensions of CK. The implementation of the calculi for extensions of CK with axioms ID and MP are very similar. For systems allowing ID, contexts are triples [Context, AppliedConditionals, AllowID]. The third element AllowID is a flag used in order to implement the restriction on the application of the rule $(I D)$, namely the rule is applied to a context only if $\mathrm{AllowID}=\mathrm{true}$, as follows: 


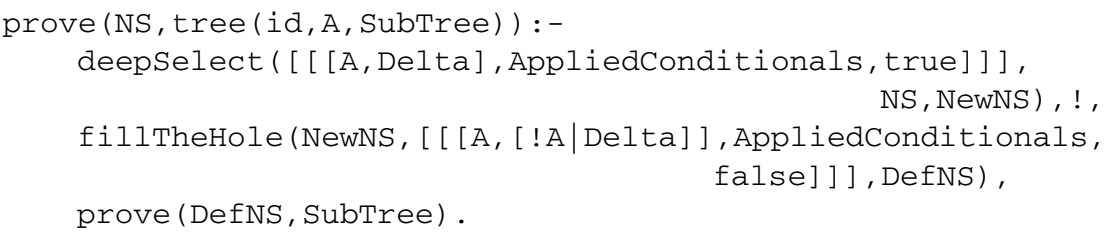

When $(I D)$ is applied to [Context, AppliedConditionals, true], then the predicate prove is invoked on the unique premise of the rule DefNS, and the flag is set to false in order to avoid multiple applications in a backward proof search.

The restriction on the application of the rule $(M P)$ is implemented by equipping the predicate prove by a third argument, AppliedMP, keeping track of the negated conditionals to which the rule has already been applied in the current branch. The clause of prove implementing $(M P)$ is:

1. prove (NS, AppliedMP, tree (mp, A, B, Sub1, Sub2)) :-

2. deepSelect $([!(A=>B)], N S$, NewNS $)$,

3. $\backslash+\operatorname{member}(\mathrm{A}=>\mathrm{B}, \mathrm{AppliedMP}), !$

4. fillTheHole (NewNS, $[A, !(A=>B)], N S 1)$,

5. fillTheHole (NewNS, [!B, ! $(A=>B)], N S 2)$,

6. $\operatorname{prove}(\mathrm{NS} 1,[\mathrm{~A}=>\mathrm{B} \mid \mathrm{AppliedMP}], \mathrm{Sub} 1)$,

7. prove (NS2, [A => B|AppliedMP], Sub2).

The rule is applicable to a formula $\neg(A \Rightarrow B)$ only if [A $\Rightarrow>\mathrm{B}$ ] does not belong to AppliedMP (line 3). When $(M P)$ is applied, then [A $=>\mathrm{B}$ ] is added to AppliedMP in the recursive calls of prove on the premises of the rule (lines 6 and 7).

The implementation of the calculus for the flat fragment of $\mathrm{CK}+\mathrm{CSO}+\mathrm{ID}$, corresponding to KLM cumulative logic $\mathbf{C}$, is similar to that for $\mathrm{CK}+\mathrm{ID}$; the only difference is that $\left(\Rightarrow^{-}\right)$is replaced by $(C S O)$. This does not make use of the predicate deepSelect to "look inside" a sequent to find the principal formulas $\neg(A \Rightarrow B)$ and $[C: \Delta]$ : since the calculus only deals with the flat fragment of the logic under consideration, such principal formulas are directly selected from the current sequent by easy membership tests (standard Prolog predicates member and select), without searching inside other contexts. Due tu space limitations, we omit details for extensions with CEM.

\section{Performance of NESCOND}

The performances of NESCOND are promising. We have tested it by running SICStus Prolog 4.0.2 on an Apple MacBook Pro, 2.7 GHz Intel Core i7, 8GB RAM machine. We have compared the performances of NESCOND with the ones of two other provers for conditional logics: CondLean 3.2, implementing labelled sequent calculi [12], and the goal-directed procedure GOALDUCK [13]. We have tested the three provers (i) on randomly generated sequents, obtaining the results shown in Figure 2 and (ii) over a set of valid formulas. Concerning $\mathrm{CK}$, we have considered 88 valid formulas obtained by translating $\mathrm{K}$ valid formulas ( $\square A$ is replaced by $\top \Rightarrow A$, whereas $\diamond A$ is replaced by $\neg(\top \Rightarrow \neg A)$ ) provided by Heuerding, obtaining the results in Figure 3 . 
Concerning (i), we have tested the three provers over 2000 random sequents, whose formulas are built from 15 different atomic variables and have a high level of nesting (10): NESCOND is not able to answer only in $0.05 \%$ of cases ( 1 sequent over 2000 ) within 10 seconds, whereas both GOALDUCK and CondLean are not able to conclude anything in more than $3 \%$ of cases (60 tests over 2000). If the time limit is extended to 2 minutes, NESCOND answers in $100 \%$ of cases, whereas its two competitors have still more than $1.30 \%$ of timeouts. The difference is much more significant when considering sequents with a lower level of nesting (3) and whose formulas contain only 3 different atomic variables: with a time limit of 10 seconds, NESCOND is not able to answer only in $9.09 \%$ of cases, whereas both CondLean and GOALDUCK are not able to conclude in $16.55 \%$ and in $51.15 \%$ of cases, respectively: this is explained by the fact that NESCOND is faster than the other provers to find 355 not valid sequents (against 17 of CondLean and 34 of GOALDUCK) within the fixed time limit.

Concerning (ii) the performances of NESCOND are also encouraging. Considering $\mathrm{CK}$, NESCOND is not able to give an answer in less than 10 seconds only in 5 cases over 88 , against the 8 of CondLean and the 12 of GOALDUCK; the number of timeouts drops to 4 if we extend the time limit to 1 minute, whereas this extension has no effect on the competitors (still 8 and 12 timeouts). We have similar results also for extensions of $\mathrm{CK}$ as shown in Figure 3. here we have not included GOALDUCK since the most formulas adopted do not belong to the fragments admitting goal-directed proofs [13].

These results show that the performances of NESCOND are encouraging, probably better than the ones of the other existing provers for conditional logics (notice that there

\begin{tabular}{|c|c|c|c|c|c|c|c|}
\hline \multirow{2}{*}{$\begin{array}{l}\text { number of } \\
\text { tests: } 2000\end{array}$} & \multicolumn{3}{|c|}{ Prop. vars: 15 , Depth: 10, Timeout: $10 \mathrm{~s}$} & \multirow{2}{*}{$\begin{array}{l}\text { number of } \\
\text { tests: } 2000\end{array}$} & \multicolumn{3}{|c|}{ Prop. vars: 15 , Depth: 10 , Timeout: $2 \mathrm{~min}$} \\
\hline & yes & no & timeout & & yes & no & timeout \\
\hline GoalDuck & $75,65 \%$ & $21,35 \%$ & $3,00 \%$ & GoalDUCK & $74,50 \%$ & $23,65 \%$ & $1,85 \%$ \\
\hline CondLean & $77,75 \%$ & $19,10 \%$ & $3,15 \%$ & CondLean & $74,90 \%$ & $23,80 \%$ & $1,30 \%$ \\
\hline NESCOND & $77,75 \%$ & $22,20 \%$ & $0,05 \%$ & NESCOND & $75,40 \%$ & $24,65 \%$ & $0,00 \%$ \\
\hline & & \multirow{2}{*}{$\begin{array}{l}\text { number of } \\
\text { tests: } 2000\end{array}$} & \multicolumn{3}{|c|}{ Prop. vars: 3 , Depth: 3 , Timeout: $10 \mathrm{~s}$} & & \\
\hline & & & yes & no & timeout & & \\
\hline & & GoalDUCK & $47,15 \%$ & $1,7 \%$ & $51,15 \%$ & & \\
\hline & & CondLean & $77,6 \%$ & $0,85 \%$ & $16,55 \%$ & & \\
\hline & & NESCOND & $73,2 \%$ & $17,75 \%$ & $9,05 \%$ & & \\
\hline
\end{tabular}

Fig. 2. NESCOND vs CondLean vs GoALDUCK over 2000 random sequents

\begin{tabular}{|c|c|c|c|c|c|c|c|c|c|c|}
\hline \multicolumn{5}{|c|}{ Timeouts for $\mathrm{CK}$} & \multicolumn{6}{|c|}{ Timeouts for extensions of CK } \\
\hline & $100 \mathrm{~ms}$ & 1s & $10 \mathrm{~s}$ & $1 \mathrm{~m}$ & & \multicolumn{2}{|c|}{$1 \mathrm{~ms}$} & \multicolumn{2}{|c|}{ 1s } & $10 \mathrm{~s}$ \\
\hline CondLean & $12,50 \%$ & $12,50 \%$ & $9,09 \%$ & $9,09 \%$ & CondLean & \multicolumn{2}{|c|}{$48,08 \%$} & \multicolumn{2}{|c|}{$36,54 \%$} & $28,85^{\circ}$ \\
\hline GoalDUCK & $21,71 \%$ & $18,26 \%$ & $13,88 \%$ & $13,88 \%$ & NESCOND & \multicolumn{2}{|c|}{$38,46 \%$} & \multicolumn{2}{|c|}{$28,85 \%$} & 26,92 \\
\hline NESCOND & $12,50 \%$ & $10,23 \%$ & $5,68 \%$ & $4,55 \%$ & & & & & & \\
\hline \multicolumn{5}{|c|}{ Timeouts for systems without CEM } & \multicolumn{6}{|c|}{ Timeouts for systems CEM and CEM+ID } \\
\hline & \multicolumn{2}{|r|}{$1 \mathrm{~ms}$} & $1 \mathrm{~s}$ & $10 \mathrm{~s}$ & & $1 \mathrm{~ms}$ & \multicolumn{2}{|c|}{ 1s } & \multicolumn{2}{|c|}{$10 \mathrm{~s}$} \\
\hline \multicolumn{2}{|l|}{ CondLean } & $19,23 \%$ & $15,38 \%$ & $13,46 \%$ & CondLean & $51,72 \%$ & \multicolumn{2}{|c|}{$37,93 \%$} & \multicolumn{2}{|c|}{$27,59 \%$} \\
\hline \multicolumn{2}{|l|}{ NESCOND } & $5,77 \%$ & $0,00 \%$ & $0,00 \%$ & NESCOND & $58,62 \%$ & & & & $8 \%$ \\
\hline
\end{tabular}

Fig. 3. NESCOND vs CondLean vs GOALDUCK: timeouts over valid formulas 
is no set of acknowledged benchmarks for them). Figure 3 shows that this also holds for extensions of CK: for systems not allowing CEM, NESCOND gives an answer in 95\% of the tests (all of them are valid formulas) in less than $1 \mathrm{~ms}$. The performances worsen in systems with CEM because of the overhead of the termination mechanism.

\section{Conclusions and Future Issues}

We have presented NESCOND, a theorem prover for conditional logics implementing nested sequent calculi introduced in [1]. Statistics in section 4 show that nested sequent calculi do not only provide elegant and natural calculi for conditional logics, but they are also significant for developing efficient theorem provers for them. In future research we aim to extend NESCOND to other systems of conditional logics. To this regard, we strongly conjecture that adding a rule for the axiom (CS) $(A \wedge B) \rightarrow(A \Rightarrow B)$ will be enough to cover the whole cube of the extensions of CK generated by axioms (ID), (MP), (CEM) and (CS). This will be object of subsequent research. We also aim at comparing the performances of NESCOND with those of CoLoSS [7], a generic-purpose theorem prover for coalgebraic modal logics which can handle also basic conditional logics.

\section{References}

1. Alenda, R., Olivetti, N., Pozzato, G.L.: Nested Sequent Calculi for Conditional Logics. In: del Cerro, L.F., Herzig, A., Mengin, J. (eds.) JELIA 2012. LNCS, vol. 7519, pp. 14-27. Springer, Heidelberg (2012)

2. Alenda, R., Olivetti, N., Pozzato, G.L.: Nested Sequents Calculi for Normal Conditional Logics. Journal of Logic and Computation (to appear)

3. Beckert, B., Posegga, J.: leantap: Lean tableau-based deduction. JAR 15(3), 339-358 (1995)

4. Brünnler, K., Studer, T.: Syntactic cut-elimination for common knowledge. Annals of Pure and Applied Logic 160(1), 82-95 (2009)

5. Fitting, M.: Prefixed tableaus and nested sequents. A. Pure App. Log. 163(3), 291-313 (2012)

6. Goré, R., Postniece, L., Tiu, A.: Cut-elimination and proof-search for bi-intuitionistic logic using nested sequents. In: Advances in Modal Logic, vol. 7, pp. 43-66 (2008)

7. Hausmann, D., Schröder, L.: Optimizing Conditional Logic Reasoning within CoLoSS. Electronic Notes in Theoretical Computer Science 262, 157-171 (2010)

8. Kashima, R.: Cut-free sequent calculi for some tense logics. St. Logica 53(1), 119-136 (1994)

9. Kraus, S., Lehmann, D., Magidor, M.: Nonmonotonic reasoning, preferential models and cumulative logics. Artificial Intelligence 44(1-2), 167-207 (1990)

10. Lewis, D.: Counterfactuals. Basil Blackwell Ltd. (1973)

11. Nute, D.: Topics in conditional logic. Reidel, Dordrecht (1980)

12. Olivetti, N., Pozzato, G.L.: CondLean 3.0: Improving Condlean for Stronger Conditional Logics. In: Beckert, B. (ed.) TABLEAUX 2005. LNCS (LNAI), vol. 3702, pp. 328-332. Springer, Heidelberg (2005)

13. Olivetti, N., Pozzato, G.L.: Theorem Proving for Conditional Logics: CondLean and GoalDuck. Journal of Applied Non-Classical Logics (JANCL) 18(4), 427-473 (2008) 